\title{
El Estado, el interés general y los grupos de interés
}

Joaquín M. Molins López-Rodó*

En el debate político, uno de los argumentos que se utilizan para valorar las propuestas de actuación de los partidos políticos, tanto los que apoyan al Gobierno como las alternativas que presenta la oposición, consiste en relacionarlas con la consecución del interés general. Se asocia la actuación de los Poderes Públicos con éste, y se rechaza cualquier medida que no pueda incluirse en dicha categoría.

Este artículo quiere ser una aportación al debate sobre la subsistencia y posible significado del concepto de interés general, a partir de los problemas teóricos que implica la participación de los grupos de interés en el proceso de decisión de las Administraciones Públicas.

El punto de partida se enmarca en el proceso de cambio producido en las relaciones entre el Estado, entendido como un conjunto de Instituciones y Administraciones, los actores políticos no institucionales, y la Sociedad. Las dimensiones y alcance del presente estudio no permiten abordar con detenimiento este aspecto, pero resulta indispensable realizar algunos comentarios para enmarcar la presente reflexión.

M. Crozier, en un artículo publicado en el no 2 de esta revista, señalaba algunos síntomas del proceso de cambio: complejidad e interdependencia de actividades, incremento de la libertad individual, la lógica del desarrollo económico y social, la mediatización de las relaciones humanas (CrOzIER 1995, págs. 94-96); y se interrogaba sobre la subsistencia del concepto clásico del interés general. En sus propias palabras thasta hace veinte años, los estadistas estaban seguros de ser capaces de definir el interés general. (Crozier 1995, pág. 97).

A. Porras, desde una perspectiva jurídica, aborda las relaciones entre Sociedad y Estado en un contexto postsocial. Señala, como elementos de cambio, la fragmentación de la realidad social que debe ser representada y la internacionalización de los problemas a resolver (Porras 1994, págs. 55-56). Coincide también con Crozier en la crisis del concepto de interés general, entendido como interés unitario y homogéneo.

En la medida que podemos establecer una vinculación entre un proceso de cambio de carácter general y la posible vigencia del interés general como concepto operativo, es por lo que creo conveniente analizar, desde la perspectiva politológica, nuestra tradición político-administrativa en lo que se refiere al impacto de dicho concepto y su evolución hasta fechas recientes. El hilo conductor lo constituirán las relaciones entre los grupos privados y las Administraciones Públicas. Más adelante, formularemos algunas propuestas sobre las relaciones entre los diferentes actores públicos y privados en el contexto del proceso de toma de decisiones de los poderes públicos.

El modelo español de Administración contemporánea está influido por la tradición francesa postrevolucionaria. La consecución del interés general se convierte en el mito fundador de la acción política y administrativa (MENY 1990). Cuando la Asamblea Nacional aprobó la Ley Chapelier (1791), que rechazaba el derecho de asociación de los grupos organizados, su principal argumento fue que entre los intereses del individuo y el interés general no había lugar para otro tipo de intereses de grupos o colectivos. A pesar de planteamientos tan radicales, ello no impidió en la práctica el mantenimiento de algunas estructuras corporativas del Antiguo Régimen.

En España, la recepción de la doctrina francesa tiene una clara influencia en nuestros primeros tratadistas -SálNZ DE ANDINO, Oljván, Colmeiro, Mellado, Santamaría de Paredes, Posada. Garcia DE ENTERRÍa, en su trabajo sobre la Revolución Francesa y la Administración Contemporánea, subraya la importancia que el concepto del interés general tiene en la construcción doctrinal de la Administración Española y destaca las afirmaciones de 
Groe-ThuYSen: :La ley cuida el interés general, y defiende el bien público contra los intereses versátiles y contradictorios de los particulares" (GARCía DE ENTERRÍA 1972, pág. 10).

La identificación entre la Administración Pública y el interés general, así como el rechazo a la intervención de los grupos privados, se mantiene prácticamente invariable en nuestra doctrina hasta fechas muy recientes, como ilustra el trabajo de A. NIETo (1991). El propio autor reconoce la ignorancia recíproca que en torno al concepto se ha producido entre la Ciencia Política y la Ciencia del Derecho, común a otros aspectos de nuestra realidad político-administrativa.

La década de los ochenta contempla una evolución doctrinal con aspectos contradictorios. Por una parte, se sigue manteniendo la doctrina tradicional: el interés general está vinculado a los fines de la Administración Pública (Santamaría PASTOR 1988, pág. 36). A su vez se producen cambios, al amparo del nuevo marco jurídico constitucional y, especialmente, a partir del reconocimiento del pluralismo político y social como un valor constitucional fundamental. Este nuevo valor comporta que el interés público sólo puede definirse normativamente por el Parlamento y, consiguientemente, la Administración carece de legitimidad autónoma para definirlo (Santamaría Pastor 1988, pág. 249).

Algún autor, como ORTEGA hàce especial hincapié èn este evolución doctrinal. En su prólogo a la obra de GianNini sobre el Poder Público (1991), critica la doctrina hegemónica hasta entonces dominante, acusándola de reducir la actividad administrativa a una única Administración y un único interés público. Frente a ello destaca la obra del profesor italiano, en cuanto es receptora de los nuevos valores del pluralismo, que comporta tanto una pluralidad de Administraciones como una pluralidad de intereses públicos. En sus propias palabras alas decisiones públicas no pueden caer en el terreno de la neutralidad inútil del interés generalı (OrTEGA 1991, pág. 10).

La corriente mayoritaria en la doctrina administrativista experimenta cambios en la segunda mitad de la década. La invocación del interés general desaparece y la preocupación doctrinal se desplaza al respeto de los derechos fundamentales en las relaciones entre los particulares y la Administración. También preocupa la posible autonomía de los decisores públicos frente a los derechos de los ciudadanos. Como subraya García DE ENTERRía -Ha concluido la invocación ritual de los intereses generales como sinónimos de superiores, tras de la cual, por cierto, suelen parapetarse tantas veces las comodidades, sino los intereses menos respetables, de la burocracia (GARCíA DE ENTERRÍA 1989, pág. 47). Según esta interpretación, los derechos fundamentales de los ciudadanos, en cuanto valor superior del ordenamiento jurídico, prevalecen incluso sobre los intereses de la Administración. Desaparece la antigua concepción de los intereses particulares como perversos, y la nueva doctrina se centra en la defensa de los intereses particulares frente a la Administración.

El cuestionamiento de la vieja concepción del interés general no sólo afecta a la relación de los ciudadanos individuales y los Poderes Públicos, sino que obliga a plantear el problema con más amplitud. Si la Administración debe responder a las demandas de los ciudadanos, ¿cómo se agregan los intereses de cada individuo y cómo llegan éstos ante las distintas Administraciones? En otras palabras, si todo tipo de intereses son igualmente defendibles ante las distintas Administraciones Públicas, ¿éstos se canalizan únicamente de forma individual o los actores - partidos políticos y grupos de interés- tienen un papel a jugar en el proceso de decisión pública?

Uno de los síntomas de cambio en el proceso de toma de decisiones por las modernas Administraciones Públicas consiste en la necesidad de implicar a los sectores afectados, estimulando su participación. Los diversos mecanismos de ésta han sido también objeto de atención por la doctrina administrativa, aunque con contenidos contradictorios. Algún autor ha señalado su preocupación por las nuevas técnicas de participación que ha desarrollado la Administración española, al considerar que pueden llegar a ser incompatibles con el principio de legalidad que sanciona nuestra Constitución (FoNT 1985). Para superar este problema, otros autores han reconocido la necesidad de redefinir los intereses jurídicos defendibles por la Administración. La vieja y tajante separación entre los intereses individuales, por un lado, y el interés general, por otro, ya no resulta operativa. Desde la óptica jurídica se ha producido la construcción de nuevas categorías jurídicas, y en particular los conceptos de interés difuso e interés colectivo. El primero consistiría en «el interés que cada individuo posee por el hecho de pertenecer a una pluralidad de sujetos" (SÁNCHEZ MORÓN 1980, pág. 117) y se diferenciaría del interés propio de una comunidad organizada. Por su parte, el interés colectivo es una especificación del interés difuso pero tampoco es el interés propio de una organización. En su argumentación, una comunidad de obreros metalúrgicos, por ejemplo, tendría un determinado interés difuso, mientras que el sindicato o sindicatos correspondientes defenderían intereses colectivos. SÁNCHEZ MORÓN reconoce que este intento de diferenciación resulta cuando menos discutible. Otro sector doctrinal sostiene que, en un ordenamiento jurídico de base pluralista, tal distinción no resulta operativa y reconoce que existen tantos intereses públicos como organizaciones.

Esta diferenciación de los intereses jurídicos tiene importncia en lo que respecta a los mecanismos de intervención de las entidades representativas de intereses en el proceso de elaboración de las decisiones públicas. En España, la Ley de Procedimiento Administrativo establecía un mecanismo formal de consulta, irrelevante en el contexto no pluralista del Franquismo. 
Sin embargo, resulta significativo que en una primera interpretación postconstitucional, sólo se consideraban incluidas en al trámite de audiencia a las Corporaciones de Derecho Público, mientras que las Asociaciones nacidas al amparo del pluralismo político y social quedaban excluidas (ReBOLo 1988). La última modificación legislativa (Ley 30/1992 art. 31.2) ha admitido en la fase de consulta formal a las nuevas organizaciones representativas de los diversos intereses sociales.

Podemos concluir esta parte afirmando que no ha sido hasta muy recientemente cuando la doctrina ha admitido la participación formal de los grupos organizados en el proceso formal de consulta. También es constatable su preocupación por las relaciones entre los particulares y la Administración, pero no se preocupa específicamente por el papel de los grupos organizados en el proceso de decisión público. En cuanto al concepto de interés general, éste ha cumplido históricamente una función básicamente legitimadora de la acción de los poderes públicos, aunque ha operado de manera ambivalente: por un lado, ha sido entendido como suma consensual de intereses particulares y, por otro, un interés que trasciende de los intereses individuales. En la actual situación, la invocación del interés general sigue utilizándose para justificar las decisiones de los poderes públicos, pero no contribuye a conocer cómo los intereses privados -individuales y colectivos- pueden influir en el proceso de decisión de nuestras Administraciones Públicas.

La participación de los grupos organizados en el sistema político constituye un tema clásico dentro de la Ciencia Política. A los efectos de este artículo, considero como grupo de interés todo grupo organizado en defensa de un interés común, con independencia de su modelo de organización y el tipo de interés que se defiende. De un modo operativo la actividad de los grupos de interés podría ser definida como aquella cuya finalidad es influir en la formulación e implementación de las políticas públicas (Grant 1989, pág. 9).

El punto de partida es que los intereses privados de carácter colectivo, independientemente de su estructura organizativa, intentan ahacerse oírn en el proceso de toma de decisiones de las Administraciones Públicas. Lo que deseamos conocer es cómo las Administraciones Públicas -responsables últimas de la decisión y por lo que responden periódicamente ante el electoradotienen más o menos en cuenta las posturas de los distintos grupos, organizados o no. Asumida la pluralidad de intereses existentes en la Sociedad, icómo se vehiculan las demandas de carácter colectivo?

Los intereses privados, independientemente de su calificación jurídica, compiten entre sí y ante las Administraciones Públicas; éstas no pueden abstraerse de la pluralidad de intereses existentes si no quieren convertirse en el temido "Leviatán" que hace caso omiso de las aspiraciones y necesidades de la Sociedad.
Desde la perspectiva politológica, el tema de la participación de los grupos privados en el proceso de decisión pública sugiere varias consideraciones. En primer lugar, el problema de las características diferenciales de los distintos grupos privados que se relacionan con la Administración. Resulta operativa a estos efectos la distinción que sobre los grupos de interés utiliza el profesor Grant. Por una parte, operan los grupos que representan a un sector de la comunidad y que persiguen su propio interés - sectional groups—. Sus posibles miembros están limitados a los pertenecientes a un sector específico de la comunidad. Por otra parte, los grupos que, unidos por una creencia común, actúan en nombre del mismo - cause groups- Estos últimos tiene una potencialidad de membership mucho mayor, pues de lo que se trata es compartir una determinada opinión. Con relación al tipo de demandas que formulan, los primeros actúan de manera específica y concreta, con un alto grado de contenido técnico y, en ocasiones, de escaso interés entre los ciudadanos, aunque no por ello menos importantes por sus implicaciones económicas y sociales. Los segundos tratan de incidir en issues más genéricos y controvertidos en el debate político. En relación a los posibles beneficiados o perjudicados por la decisión, en el primer grupo afecta directamente a colectivos definidos, mientras que en los segundos los posibles beneficios tienen un carácter más difuso. Desde el punto de vista de la Administración, los diferentes tipos de grupos y sus demandas - concretas o genéricas - tienen un mismo objetivo: ser tenidos en cuenta en el proceso de la toma de decisiones.

En segundo lugar, las relaciones "privilegiadas" que se establecen entre ciertos grupos privados y la Administración. Aunque el tema lo he desarrollado más ampliamente en otro trabajo (Mousns 1994, cap. 2), quisiera exponer aquí algunas conclusiones en lo que respecta a la situación española. El sistema resultante supone una cierta "complicidad. entre el Estado y algunos grupos organizados. Por una parte, las Administraciones otorgan una serie de privilegios a organizaciones que se proclaman privadas e independientes; éstas, a su vez reclaman la ayuda de la Administración para conseguir afiliados - caso de los Colegios profesionales-, para mantenerse económicamente- sindicatos y patronales "más representativas", o ambas cosas a la vez - Cámaras Oficiales-. Parece existir una cierta desconfianza en la actuación del ciudadano respecto a su capacidad y voluntad de adherirse o no a las organizaciones que crea conveniente, y contribuir a su sostenimiento económico. También existe una cierta prevención a la actuación de organizaciones privadas, basadas en el pluralismo social y la libertad de asociación, y la tendencia a sustituirlas o hacerlas coincidir con otro tipo de organizaciones con un cierto status público.

El tercer aspecto a considerar son las relaciones que en los sistemas políticos pluralistas se establecen entre los actores privados y las Administraciones Públicas, y sus canales de influencia mutua. Aunque lo que sucede en España no es muy diferen- 
te a lo que ocurre en otros sistemas políticos, el reconocimiento explícito de la influencia de los grupos de interés es muy diferente. La explicación de este fenómeno es compleja y las causas son múltiples; podrían mencionarse el funcionamiento del sistema representativo y el papel de los partidos políticos, la tradición administrativa y la vigencia del concepto de interés general, así como cuestiones relacionadas con la cultura política.

En la dificultad de reconocimiento de la actividad de presión ha influido también, en los últimos años, la polémica sobre la corrupción y la proliferación de actividades calificadas genéricamente como "tráfico de influencias". ¿Cómo distinguir la intermediación entre los intereses de los distintos grupos sociales y las Administraciones Públicas, respetando la autonomía de decisión de esta última para conseguir beneficios privados? El criterio diferenciador de ambas actividades podría establecerse en que en las primeras se reconoce a las Administraciones Públicas su condición de mediadora entre los diferentes intereses en presencia, mientras que en las segundas lo que se busca es obtener un favor -individual o colectivo- mediante una contraprestación económica. Ejemplos del primer tipo de actividades los constituirían las políticas públicas de carácter regulativo, mientras que en las políticas distributivas resulta más difícil establecer una clara línea de separación entre ambos tipos de actividades. En todo caso, podrían sugerirse algunas medidas para separar la actividad de influencia de las prácticas ilegales. En primer lugar, clarificar las reglas de juego en lo referente a las relaciones entre los grupos privados y las Administraciones. Las ambigüedades y contradicciones de la actual legislación española provocan que, por una parte, se mantengan barreras de acceso a los grupos en sus relaciones con las Administraciones Públicas mientras que, al mismo tiempo, la propia Administración solicita el concurso y la colaboración de los grupos privados para el desarrollo de las diferentes políticas públicas (SuBiraTs 1992). En segundo lugar, establecer una regulación de la propia actividad de presión. La presentación, en el mes de diciembre de 1992, de una proposición no de ley para regular la actividad de los grupos, no tuvo el eco político y académico que el tema requiere, desapareciendo de las prioridades legislativas de la actual legislatura. En este aspecto, podrían ser tenidas en cuenta las experiencias más recientes de Canadá (Pross. 1991) o del Parlamento Europeo (Greenwwood et alt. 1995). En tercer lugar, debe producirse una mayor delimitación de actividades y responsabilidades en los traspasos de personal entre el sector privado y las Administraciones Públicas, ya que afecta directamente a las normas de actuación de los lobistas profesionales.

Desde este punto de vista, la publicidad y la transparencia ante la opinión pública de las relaciones entre los distintos grupos de interés y las Administraciones constituyen la garantía de que, en un sistema político democrático y pluralista, la actividad de los grupos privados no sustituye a la voluntad de los ciudadanos expresada a través del sufragio e interpretada por las instituciones representativas. En palabras de Grant (1989 pág. 163) el poder de los grupos está basado, más que en tomar decisiones, en su habilidad para persuadir e influenciar a las Administraciones.

- Profesor de Ciencia Política. UAB.

\section{Bibliografia}

Crozier, M. (1995) -La posición del Estado ante los otros actores. Gestión y Análisis de Politicas Públicas n. 2.

FONT, T. (1985) -Organos consultivos. Revista de Administración Pública n. 108. GarCía de EnterRia, E. (1972) Revolución Francesa y Administración Contemporánea. Taurus. Madrid.

GarCIA DE ENTERRIA, E. (1989) Hacia una nueva justicia administrativa. Civitas. Madrid.

Grant, W. (1989) Pressure groups, Politics and Democracy in Britain.Philip Allan. London.

GrEenwOOD, J. Y MCLAUGHIN, A. (1995) . The management of interest representation in the European Union. Journal of Common Market Studies, vol. 33.1.

MeNY, Y. (1990) -Los grupos de interés en Francia. Revista del Centro de Estudios Constitucionales, n. 7.

Mouins, J. (1994) -Los grupos de interés en España. Papeles de la Fundación para el Análisis y los Estudios Sociales, n. 7.

NIETo, A. (1991) -Los intereses generales. Homenaje a E. Garcia de Enterria. Tomo III. Civitas. Madrid
ORTEGA, L. (1991) •Prólogo• a GiAnNINI, S. El poder püblico: Estados y Administraciones Püblicas. Civitas. Madrid.

Porras, A. (1994) -Representación y democracia avanzada. Cuadernos y Debates del Centro de Estudios Constitucionales, n. 50.

Pross, P. (1991) .The rise of the Lobbying issue in Canada: the Business Card Bill - en Jordan, G. (ed) The commercial Lobbyists. Aberdeen University Press. Aberdeen.

ReBollo, M. (1988) -La participación de las entidades representativas de intereses en el procedimiento de elaboración de disposiciones administrativas generales. Revista de Administración Pública n. 115.

SánChez Morón, M. (1980) la participación del ciudadano en la Administración Pública. Centro de Estudios Constitucionales. Madrid.

Santamaría Pastor, J.A. (1988) Fundamentos de Derecbo Administrativo. Centro de Estudios Ramón Areces. Madrid.

Subirats J. (1992) . Un problema de estilo. La formación de Políticas Públicas en España. Cuadernos y Debates del Centro de Estudios Constitucionales, n. 38. 